Fall 3: Mutter, 30 Jahre, im dritten Monat schwanger, Impfung gegen MMR und Varizellen nicht erinnerbar, nach eigenen Angaben Masern/Mumps durchgemacht, Röteln und Windpocken nicht. Ihr 18 Monate altes Kind soll vor Kita-Aufnahme eine erste Dosis MMR/Varizellen-Impfstoff erhalten, anamnestisch keine der Krankheiten durchgemacht.

Frage: Kann das Kind die Impfung erhalten? Antwort: Ja, kein Problem. Beim RötelnImpfstoff ist eine nasopharyngeale Ausscheidung häufig, aber es kommt zu keiner Erkrankung bei Kontaktpersonen. Auch für Masern- und Mumps-Impfviren ist keine Übertragung vom Impfling auf Kontaktpersonen nachgewiesen. Kinder einer seronegativen Schwangeren können also ohne Bedenken geimpft werden. Bei Varizellen gibt es einen dokumentierten Fall einer Übertragung des Impfvirus auf eine schwangere Kontaktperson - ohne Komplikationen, wie Prof. Dr. Fred Zepp aus Mainz berichtete. Das Risiko eines fötalen Varizellen-Syndroms nach Infektion der Schwangeren mit WildVirus liegt bei $0,4-2 \%$, das Risiko eines Kontakts mit dem Wildvirus durch das ungeimpfte Kita-Kind ist höher als das theoretische Risiko durch das Impfvirus.

Fall 4: Donnerstagnachmittag, Jugendsprechstunde: 17-jährige Internatsschülerin mit fieberhaftem Infekt und beginnendem Ausschlag. Sie berichtet, der Infekt ginge seit Wochen im Internat herum. Eine Maserninfektion ist klinisch nicht auszuschließen.

Frage: Wie gehen Sie weiter vor?

Antwort: Masern seien heute eine Infektion Jugendlicher und junger Erwachsener, die selten und klinisch nicht immer eindeutig zu diagnostizieren ist, erklärte Leidel. Bei Verdacht müsse schnell reagiert werden, um einen Ausbruch möglichst im Keim zu ersticken. Also: Serologie abnehmen, schon bei Verdacht auf Masern eine Meldung an das Gesundheitsamt vornehmen, Kontaktpersonen für eine Postexpositionsprophylaxe (PEP) ermitteln. Ziel der Maßnahmen ist die Eradikation der Masern, die bislang nicht gelungen ist. 2011 gab es hierzulande bereits über 1.500 Fälle.

Fall 5: Drei Monate altes Kind, unerwartete und in dieser Form bisher nicht gesehene, körperliche Reaktion nach Impfung.

Frage: Wie gehen Sie bei Verdacht auf eine Impfkomplikation weiter vor?
Antwort: Bei entsprechendem Verdacht besteht die Pflicht einer namentlichen Meldung beim Gesundheitsamt, das dann beim weiteren Vorgehen Unterstützung bietet. Das Meldeformular kann beim Gesundheitsamt oder beim Paul-Ehrlich-Institut heruntergeladen werden. Das gleiche Formular kann auch zur pflichtgemäßen Meldung bei der Arzneimittelkommission der deutschen Ärzteschaft (Akd̈̈), allerdings in anonymisierter Form, verwendet werden. Zur Meldung beim Impfstoffhersteller besteht keine Pflicht, die Experten halten sie jedoch für sinnvoll. Schwierig sei mitunter die Abgrenzung zwischen üblichen Impfreaktionen und darüber hinaus gehenden Komplikationen, bemerkte Leidel. Hier biete der Meldebogen Hilfestellung.

Fall 6: Säugling, vier Monate alt, Verdacht auf Rotavirusinfektion bei akuter Gastroenteritis mit Durchfall und Erbrechen. Nach Impfung mit Rotarix im Alter von zwei Monaten stünde jetzt die zweite Impfung an.

Frage: Impfen wie geplant? Stuhldiagnostik abwarten? Oder später impfen?

Antwort: Eine akute Gastroenteritis ist laut Fachinformation eine Kontraindikation für die Impfung, sie muss auf einen späteren Termin verschoben werden, stellte Terhardt klar. Eine Stuhluntersuchung ist nicht erforderlich. Bei dem späteren Impftermin ist auf das Impffenster zu achten: Laut Zulassung sollte die Impfserie bei Rotarix ${ }^{\circledast}$ bis zum Alter von 24 Wochen, bei Rotateq ${ }^{\circledR}$ bis Woche 26 abgeschlossen sein. Laut Terhardt hat man in den USA das Zeitfenster inzwischen erweitert, weil die Frist zum Beispiel durch Erkrankungen der Säuglinge häufig nicht immer einzuhalten ist.

Fall 7: Kind aus Afghanistan, 8 Jahre, keine Impfdokumente, keine Klärung möglich, ob es jemals geimpft wurde.

Frage: Wie gehen Sie bezüglich der Impfung mit Tetanus, Diphtherie, Pertussis vor?

Antwort: Der Kombinationsimpfstoff TdaP (Tetanus, Diphtherie, Pertussis) ist für die Grundimmunisierung nicht zugelassen. Ohne Off-Label-Gebrauch sei daher eine STIKO-konforme Impfung des Kindes nicht durchführbar, so Leidel. Grundsätzlich ist die Grundimmunisierung mit dem Mehrfachimpfstoff aber möglich, da die antigene Zusammensetzung identisch ist mit der zugelassener Impfstoffe, etwa hinsichtlich der Td-Komponente. Die STIKO arbeite derzeit daran, TdaP als Empfehlung durchzusetzen und eine entsprechende Leitlinie zu formulieren. Bis dahin muss der Off-Label-Use gut dokumentiert und entsprechend aufgeklärt werden.

Michael Koczorek

Die STIKO stellt sich: Schwierige Impffragen in der kinderärztlichen Praxis.

\title{
Wie gefährlich ist die Spritze auf dem Spielplatz?
}

Etwa 10-15\% der Drogenabhängigen, die sich ihren Stoff intravenös applizieren, sind HIV-positiv. Trotzdem geht von Spritzen, die auf der Straße oder dem Spielplatz zurückgelassen werden und mit denen sich Kinder stechen, keine Gefahr aus: „In den letzten 25 Jahren ist keine einzige HIV-Übertragung durch solche Nadelstichverletzungen bekannt geworden", erklärte PD Dr. Ulrich Baumann aus Hannover. Die Nadeln seien kaum infektiös, da sie meist schon eine Weile rumliegen. In dieser Zeit ist das Blut geronnen. Und ist es erst einmal geronnen, kommt es praktisch nicht mehr in den Körper des verletzten Kindes hinein. Jedoch Im Gegensatz dazu ist das Risiko einer Infektion mit Hepatitis B und C durchaus vorhanden. „Deshalb hier bitte den Impfstatus überprüfen, genauso wie den von Tetanus", sagte Baumann. Den

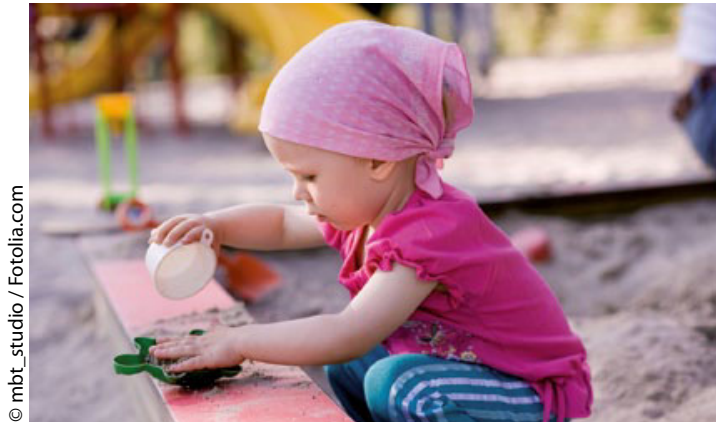

Eltern kann außerdem angeboten werden, Titerkontrollen von Hepatitis B und C durchzuführen.

$n z$

Baumann U. Aktuelle Fragen zu Nadelstichverletzungen und risikoreichem Sexualverhalten von Jugendlichen. 